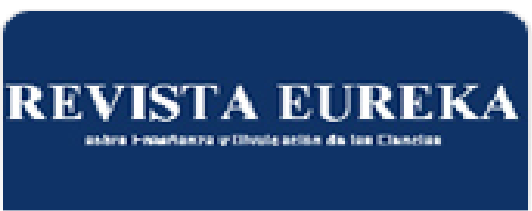

Revista Eureka sobre Enseñanza y Divulgación de las Ciencias

E-ISSN: 1697-011X

revista@apac-eureka.org

Asociación de Profesores Amigos de la

Ciencia: EUREKA

España

Sánchez Vázquez, María Alejandra

La confianza y las instituciones de divulgación: un valor fundamental para una cultura científica Revista Eureka sobre Enseñanza y Divulgación de las Ciencias, vol. 5, núm. 1, enero, 2008, pp. 2-14

Asociación de Profesores Amigos de la Ciencia: EUREKA

Cádiz, España

Disponible en: http://www.redalyc.org/articulo.oa?id=92050102

Cómo citar el artículo

- Número completo

- Más información del artículo

- Página de la revista en redalyc.org

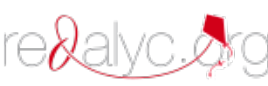

Sistema de Información Científica

Red de Revistas Científicas de América Latina, el Caribe, España y Portugal Proyecto académico sin fines de lucro, desarrollado bajo la iniciativa de acceso abierto 


\title{
LA CONFIANZA Y LAS INSTITUCIONES DE DIVULGACIÓN: UN VALOR FUNDAMENTAL PARA UNA CULTURA CIENTÍFICA ${ }^{1}$
}

\author{
María Alejandra Sánchez Vázquez² \\ Instituto de Investigación y Desarrollo Educativo \\ Universidad Autónoma de Baja California \\ sanchez_vazquez@uabc.mx, http://iide.ens.uabc.mx/
}

[Recibido en Septiembre de 2007, aceptado en Noviembre de 2007]

\section{RESUMEN ${ }^{\text {(Inglés) }}$}

A partir del análisis de dos eventos que sucedieron en un centro de ciencias en una ciudad mexicana, se invita a reflexionar sobre la necesidad de generar confianza entre los visitantes a instituciones de divulgación. Se describen los procesos de comprensión de un niño preescolar y un grupo de adolescentes en situaciones emocionales significativas, al mediar el miedo y la desconfianza entre los escuchas y los emisores del conocimiento. Se describe que el confiar en el emisor de las explicaciones científicas es un recurso ético y político necesario para fomentar una cultura científica centrada en la comprensión de los procesos de producción de conocimiento.

Palabras clave: Etnografía; centro de ciencias; comprensión; confianza; divulgación; comunicación pública de la ciencia.

\section{INTRODUCCIÓN}

Poco se habla de la importancia de la generación de confianza en las salas de museos, centros de ciencia y actividades de divulgación en general. Esta situación es relevante desde diferentes ángulos. Por ejemplo, es común hoy considerar la opinión científica como la más confiable en contraste con las creencias de la gente, sus opiniones o sus intuiciones. ¿Cómo se ha llegado a este consenso casi general en las sociedades contemporáneas? En otro sentido, la devaluación de la confianza de la gente en sí misma y sus posibilidades de comprender la ciencia es un factor clave en la orientación vocacional de los estudiantes. ¿Cómo se ha minado la confianza en la comprensión de la matemática, por ejemplo? Y ¿qué tan importante es la generación de confianza entre la gente para lograr que más personas se interesen por las ciencias y lo que éstas tienen que decir sobre el universo? Con tan sólo echar un vistazo en el tema de la confianza se percibe que se trata de un valor fundamental para comprender el papel de las ciencias en la sociedad, lo mismo que para comprender el aprecio por las versiones científicas del conocimiento humano. 
En el presente artículo se describen dos situaciones en las que diversos individuos, visitantes a un centro de ciencias, desarrollaron cierta confianza en sus interlocutores y paralelamente en su capacidad para comprender los eventos explicativos que vivieron en el centro. Con el objetivo de proponer que la generación de confianza hacia las instituciones de divulgación de las ciencias bien puede sentar las bases para generar la confianza hacia la ciencia en general y hacia sus comunidades de científicos, en este artículo se destaca la importancia que la confianza parecía tener en las situaciones evidenciadas. Si mi explicación resulta convincente para el lector, entonces la sugerencia es considerar la generación de la confianza como un elemento fundamental en la operación de salas de centros de ciencias o de cualquier institución o evento en que se divulgue conocimiento científico.

\section{UNA ETNOGRAFÍA DE LA DIVULGACIÓN CIENTÍFICA}

Las observaciones que aquí se exponen provienen de una investigación mayor realizada desde el campo de la antropología social (Sánchez Vázquez 2005). Desde septiembre de 2001 a septiembre de 2002 se hizo trabajo etnográfico en un planetario-centro de ciencias. La mayoría de la información sobre la que se fundamentó el análisis provino de la observación y la participación en la vida cotidiana en el centro, y el registro diario de descripciones sobre eventos mayores y menores, la observación de la interacción del público con los interactivos y la interacción diaria de los trabajadores del centro. Para fortalecer las descripciones generadas, también se recogieron datos en entrevistas a profundidad, entrevistas semi-estructuradas al público y a los empleados de la institución.

La primera decisión en el campo fue optar por la observación de las relaciones de los trabajadores entre sí, con el ambiente y con el público. Se descartó, en principio, hacer un estudio sobre los visitantes. La premisa para tomar esta importante decisión fue una idea sugerente de la divulgadora mexicana Julieta Fierro (2002). Ella ha hablado de la óptima posibilidad de comprender las ciencias de la misma forma en que se aprende un idioma extranjero cuando se vive en el país donde éste se habla: La inmersión de un individuo en un ambiente en donde cotidianamente se habla en alemán, por decir algo, hace más fácil y realista la posibilidad de usar correctamente y más rápido el nuevo idioma. Un centro de ciencias, donde el trabajo diario era la divulgación de las ciencias, podía ser el equivalente para el grupo de individuos que trabajaba ahí día a día; un ambiente donde el tema común era el conocimiento científico. Es decir, la prioridad sería la exposición cotidiana de ciertos individuos a la información científica y no el estudio de las visitas esporádicas de los diversos públicos. Las dos viñetas que se presentan aquí tratan, sin embargo, los procesos de comprensión de visitantes al centro. Su importancia radica en que, a partir de su análisis, es posible ahondar en la vida social del centro.

Para el análisis general de los datos se procedió organizando la información en función de temas definidos como prioritarios: el uso del espacio, la descripción de los objetos, la descripción de las interacciones entre todos los individuos-trabajadores, visitantes o empresarios relacionados-, las relaciones de poder y las negociaciones para privatizar el centro. 
Un capítulo del trabajo original (Sánchez Vázquez 2005) -que es la base del presente artículo- se dedicó al fenómeno de la comprensión del conocimiento, y éste se analizó en términos del uso y control del tiempo concedido a los individuos para entender información nueva. A partir de la observación de procesos de comprensión y del tiempo que les tomaba a los diferentes individuos comprender, emergió una relación significativa: la variabilidad del tiempo necesario para lograr la explicacióncomprensión de conocimiento nuevo - científico o no- en relación directa con el tiempo necesario para permitir el desarrollo de la confianza del individuo escucha en el individuo que explicaba.

Durante el año que duró el trabajo de campo, fui testigo de muchos eventos de interacción. Para analizar los procesos de comprensión, seleccioné dos eventos que desde mi perspectiva fueron los más significativos. Las situaciones que se describen a continuación me parecieron cruciales porque en ellas percibí la importancia que tenía el confiar en aquella persona o personas que tomaban control de la explicación de los contenidos. En la mayoría de los eventos observados - los cuales no serán descritos a profundidad en esta ocasión- las interacciones se aproximaban más a la interacción tradicional tan cuestionada entre maestro y alumnos; es decir, el que fungía como maestro/guía/emisor hablaba y quien actuaba como escucha/alumno/receptor escuchaba. La relación era unidireccional, repetitiva, masiva y recurrente. En los dos eventos que se retratan aquí, por el contrario, el contexto y la extensión de las interacciones me permitieron observar el proceso de comprensión que no había quedado expuesto en la mayoría de las interacciones entre guías, maestros y niños. Considero estos eventos como excepcionales porque los aprendices expresaron cierta reserva y prudencia frente a las aseveraciones de los emisores del conocimiento. Las dos viñetas han sido seleccionadas para describir la importancia de la participación y el desarrollo de la confianza en el proceso de comprensión.

La primera viñeta trata la reacción de un niño de preescolar en una sala de robots dinosaurios; en la segunda se describen los desafortunados eventos que se dieron en una sesión de hipnotismo entre adolescentes. Ambos sucesos tuvieron lugar en el centro de ciencias estudiado y resultaron significativos porque, como se ha mencionado ya, el proceso de comprensión fue en cierto sentido accesible a la observación. Esta institución, conocida por la población de aquella ciudad como "el planetario", era un gran centro de ciencias subdividido en varias salas donde se exponían temas científicos o tecnológicos como astronomía, una colección de automóviles de diferentes épocas, geología, física recreativa, la historia de las telecomunicaciones, un escenario geodésico para la proyección del cielo nocturno y videos de ciencias, y una sala donde se exhibía una representación del mundo jurásico. Estos dos casos se han extraído de la etnografía que en general describe cómo una institución que descuida el trabajo cotidiano y la comunicación entre sus empleados puede llegar al punto de generar una cultura de indiferencia hacia el conocimiento científico más que de aprecio por las ciencias.

\section{Ismael y los dinosaurios}

Un niño de 4 años, Ismael, es llevado junto con su grupo preescolar a una visita al planetario-centro de ciencias de la ciudad. Recorrerán las salas de la exposición del 
mundo jurásico: una serie de escenas de dinosaurios robots. A pesar de que su maestra y sus compañeros intentan convencerle de que está a salvo, Ismael se niega de todas las maneras posibles a entrar en la sala. La maestra debe atender al grupo, de manera que le pide a una guía del centro de ciencias que se encargue de Ismael mientras el resto del grupo recorre la exhibición. La guía llega a una reunión de trabajo en la sala contigua con este pequeño niño secando sus lágrimas. De varias maneras, el jefe de servicios al público y otra guía intentan persuadirle de entrar a la exhibición. Ismael va y viene secando sus lágrimas de nuevo y visiblemente más aterrado por los rugidos que llegan del fondo. Como el personal estaba en plena reunión de trabajo, me ofrezco para cuidar a Ismael y también me doy a la tarea de ayudarle a superar su miedo. De muchas maneras hago uso de mi experiencia adulta para lograr que el niño gane confianza y se atreva a entrar a la exhibición: hago un dibujo, le ofrezco quedarme con él todo el tiempo, le hablo de la irrealidad de los robots, incluso pienso en hablarle de la extinción, pero me doy cuenta de que se trata de información totalmente irrelevante para su edad y su conflicto primordial: el miedo a que los dinosaurios le hagan daño, que se coman su sándwich. En ningún momento hay espacio para bromas, el pequeño Ismael está genuinamente aterrado y la temperatura de su mano y su rostro pálido y lloroso son la evidencia. Finalmente logro que nos acerquemos a la entrada para ver desde el umbral al primer robot: un cachorro triceratops. De ahí avanzamos a la siguiente sala donde hay otros especimenes sólo moviendo colas o extremidades. Los rugidos continúan y el volumen crece ante nuestra proximidad. El miedo cede paso a la curiosidad cuando pasa un mecánico y le explica a Ismael por qué no ha de tener miedo: "Porque si yo los desconecto se detienen-como tu bicicleta ¿es que tu bici come algo?" pregunta el mecánico. "No" responde muy tímido Ismael. Finalmente, al tratar de vencer su miedo totalmente y correr a través de la peor sala, la más oscura y violenta porque dos sanguinarios dinosaurios pelean, Ismael se arrepiente y me hace salir corriendo por donde entramos. Tuvo más que suficiente. En ese momento llega su maestra y le cedo su manita friísima. Ella se sorprende ante la genuina sensación de temor.

Al analizar esta escena a posteriori noté la larga sesión de eventos que se dieron, todos con el fin de lograr que Ismael entrara en una sala de robots. Leyendo la literatura sobre el desarrollo cognitivo infantil y la socialización de los niños como partícipes en el mundo social, encuentro la descripción de Briggs (1998). Esta autora explica que la comprensión moral infantil va acercándose a la comprensión adulta a través de su participación en pequeños dramas (o escenificaciones casi teatrales) consecutivos. En estos dramas-que muchas veces son "puestos en escena" por los adultos-de a poco se va dando el cambio en la percepción infantil y el perfeccionamiento de su participación en el mundo adulto.

Analizados en independencia, cada drama con los dinosaurios tuvo como eje no sólo el objetivo de lograr que Ismael entrara a la sala o que perdiera su miedo; cada interacción llevó a todos los actores a lograr la confianza de Ismael en los adultos, en el ambiente "inofensivo" o en la inocuidad del robot en sí. Se trató de una larga sucesión de actos en los que Ismael fue desarrollando una sensación de confianza, incluso en sí mismo. Los niños llegan a comprender lo que los adultos desean que entiendan a través de su participación en dramas consecutivos y repetitivos. Es así 
porque regularmente se pide al niño que abandone la explicación inicial y empírica que se da a sí mismo para adoptar la explicación adulta de las cosas (Toren 1993, 473). Como adultos pedimos a los niños que comprendan las cosas que queremos como parte de un ciclo similar al ya vivido por nosotros (Briggs 1998).

Las implicaciones que este evento tiene para el museo de ciencias pueden ser vistas como insignificantes porque finalmente fue sólo un niño, en un grupo de treinta más, el único que enfrentó el problema. Sin embargo, no se ha de dejar pasar por alto el hecho de que cada individuo interpreta, de acuerdo a su momento, lo que se ofrece en una sala y que si se quiere capitalizar cualquier elemento para la enseñanza de las ciencias, la ficción, o el miedo, pueden ser un gran obstáculo. Baste por ahora decir que la confianza es necesaria para comenzar a comprender; y la confianza no se genera tan sólo discursivamente, sino a partir de la "puesta en escena" de situaciones que van llevando al niño a perfeccionar cierta percepción de las cosas más cercana a lo que es la visión adulta generalizada.

\section{El hipnotizador}

Este segundo evento tuvo lugar durante un evento especial anual: el Día del Sol en el planetario-centro de ciencias. Se festejaba el solsticio de verano y diversas personas fueron convocadas para el festejo. Las organizadoras-dos administradoras del centro -invitaron a estudiantes de física para dar demostraciones sobre las propiedades de la luz; a ingenieros para explicar la importancia y el uso de la energía solar en la cocción de alimentos y calentamiento del agua; a un practicante de yoga para explicar ejercicios de respiración y relajación con la ayuda de la energía solar; a danzantes creyentes en la herencia mexica y sus ritos y veneraciones a los cuatro vientos, al sol, a Quetzalcóatl-la mítica serpiente emplumada, deidad prehispánica. El evento en general era una constelación paradójica. Fue organizado por una bióloga y una administradora que optaron por un festival cultural más que por una feria de ciencias. En este evento de yuxtaposiciones, la atracción principal del día sería la sesión de un hipnotizador famoso en la ciudad.

Llego tarde al evento principal por mi renuencia a observar algo que no tiene que ver con mi misión principal, la divulgación de las ciencias. Los doscientos y tantos asientos del auditorio están ocupados ya por adolescentes inquietos que disfrutan al máximo del espectáculo. Ocho estudiantes, hombres y mujeres, todos de secundaria, han sido hipnotizados y detectados de entre la gente en el auditorio. El hipnotizador los ha invitado a pasar al escenario y a cada uno le ha llevado a hacer un número breve que hace soltar la carcajada de los espectadores. Para terminar les da instrucciones moralistas sobre cómo han de comportarse con padres y maestros. Les pide que sean trabajadores y buenas personas.

Antes de decirles que pueden despertar, el hipnotizador se toma la libertad de usar nuevamente el micrófono y su experiencia para ordenarles con una fanfarria que es momento de bailar seeeeeexy! Conforme sube el volumen de la música, pide a las chicas hipnotizadas que bailen en el escenario y a los jóvenes les ordena bajar a bailar con el auditorio. El espectáculo es agradable porque los adolescentes disfrutan bailando con mucho sentido de la seducción hacia el auditorio, incitando, bailando como estrellas pop; pero es desagradable también porque lo hacen inconscientemente 
y hay risas, bromas y euforia a su costa. Los observadores sabemos que no bailan en plenitud de consciencia y el resultado es morboso.

De pronto, el altísimo volumen de la música pop baja hasta el silencio y el hipnotizador les ordena despertar. Un joven obeso que había comenzado a abrir su pantalón pregunta genuinamente alarmado "¿Qué me hicieron?". Se escucha la carcajada del auditorio y el hipnotizador le "recuerda" que él mismo desabrochó su pantalón hacía unos minutos. Cuando los hipnotizados, ya conscientes, regresan a sus asientos, sus compañeros no pueden esperar para preguntarles qué sintieron. Al descubrir que verdaderamente no saben qué hicieron, no lo recuerdan y no saben por qué o cómo pudieron comportarse como lo hicieron, los rostros de los hipnotizados comienzan a reflejar duda, temor, incredulidad, nerviosismo y vergüenza.

Afuera en el jardín, me acerco a uno de los chicos participantes. Él me dice que cree en lo que le han contado que hizo porque conoce a sus amigos y sabe que lo conocen bien; reconoce que hay relación entre su comportamiento habitual y lo que al parecer hizo inconscientemente. Además de conocerles, son sus amigos, confía en ellos como para creer en lo que le cuentan, aunque en realidad no pueda creer que sucedió, ni comprender cómo sucedió. Todos los hipnotizados parecen sentirse igualmente perturbados. La mayoría de ellos resultan ser, por coincidencia, los mejores estudiantes de cada grupo, así que sus maestras están también algo eufóricas y sorprendidas por haber sido testigos de una transformación en esos jóvenes usualmente retraídos o normalmente discretos. Una de ellas incluso confiesa creer que la experiencia le hará bien a sus dos estudiantes hipnotizados.

El evento del hipnotizador puede parecer descabellado al tratarse del análisis de un centro de ciencias. Sería común anticipar sin dudar que en un centro de ciencias se tratarán temas relacionados con la naturaleza, con las ciencias experimentales, con la física, su matemática, o la química, más no esperaríamos un evento como el descrito aquí en tal institución. Sin embargo sucedió así y tres secundarias de la ciudad fueron invitadas y pagaron para ver ese espectáculo en la fiesta del día del Sol del único centro de ciencias en esa población. Soy de la opinión que eventos como éste no han de ser suprimidos del registro sólo porque no van de acuerdo a lo que los contenidos científicos mandan. De hecho, el que haya sucedido algo así, y muy posiblemente suceda en muchos otros centros del mundo, nos debe llamar la atención como analistas.

El factor más notable que incrementa la pertinencia de la consideración de este evento es el abuso de confianza en un contexto de educación informal y divulgación científica.

La responsabilidad frente a este desafortunado evento está diluida entre los participantes involucrados-desde el hipnotizador hasta el director del centro de ciencias, pasando por las maestras, las organizadoras, los amigos de los hipnotizados y yo misma como observadora y participante. Algo relevante de este evento, sin duda, es que los jóvenes hipnotizados accedieron a experimentar algo que se les ofreció en un sitio que tiene cierta aura de respetabilidad. Aceptaron el ejercicio, pero cuando fueron hipnotizados lo que comenzó a ser relevante para ellos no era relevante para el resto del público, por lo que fue fácil reír ante sus acciones, dada la aparente normalidad con la que actuaron extravagantemente. Siguiendo a Waterfield (2002): 
En la hipnosis, la experiencia se percibe y fluye como parte de un sueño, sin esfuerzo, con cierta ausencia de lógica-llamada la lógica de trance-en la que el hipnotizado no da mucha importancia a situaciones anómalas, aunque el público sí se la dé-lo que es la clave del éxito de estos espectáculos. En la literatura sobre hipnosis se asegura que una persona hipnotizada no hará algo en contra de su voluntad, y que de hecho, la posibilidad de la hipnosis se cimienta en la duda que enfrenta el sujeto al estar en una disyuntiva: ¿qué pasa si le hago caso y me dejo llevar?... no, tal vez sea mejor que no lo haga...¿ipero qué pasaría si hago el experimento?... no, seguro que nada sucederá... Hasta que el individuo se encuentra en un estado de semi-consciencia (Waterfield 2002, p. 26). De manera que podríamos decir que incluso los hipnotizados son responsables de lo sucedido porque en cierto grado accedieron a la hipnosis.

Este evento es significativo porque sucedió en un centro de ciencias, porque se abusó de una conducta natural humana en la que se enfrenta una disyuntiva, e incluso se abusó de una conducta que podríamos llamar científica porque los estudiantes se prestaron a experimentar, como se espera que se proceda en la investigación en ciencias. El que la hipnosis sea o no un tema relevante para un centro de ciencias no está en discusión aquí; el hecho es que un espectáculo abusivo llegó a formar parte de los eventos especiales de este centro de ciencias. Ni la institución, ni las guías, las organizadoras, y mucho menos el hipnotizador se responsabilizaron por dar una explicación de lo sucedido a los estudiantes. Una explicación relevante se ignoró. El centro no se hizo responsable de explicar lo sucedido para generar conocimiento. $Y$ aunque la posibilidad de que un evento tan cuestionable se aprovechara con fines pedagógicos habría sido aún más irritante, es igualmente perturbador el que el público de esa mañana haya dejado el centro sin explicación alguna. Creo que aquellos jóvenes aprendieron que la hipnosis es cierta y posible; tal vez estén por siempre interesados en las posibilidades de la hipnosis, pero dudo que esa sea una técnica de enseñanza-aprendizaje que se quisiera fomentar en estas instituciones.

\section{CREER PARA COMPRENDER}

Con el apoyo de la primera viñeta se concluyó que la confianza es necesaria para comenzar a comprender. Ismael parecía resistirse a entrar, a comprender que no había peligro real; sin embargo ante la posibilidad excepcional de percibir poco a poco cómo sus premisas se iban derrumbando logró al menos ver un par de robots. Para lograr comprender, Ismael tuvo que participar por sí mismo en una serie de dramas (Briggs, 1998). El tiempo que se tomó este pequeño fue un tiempo necesario para desarrollar cierto nivel de confianza.

En la segunda viñeta, los adolescentes también participaron en una serie de dramatizaciones que los ridiculizaron primero y después les dejaron expuestos a un regaño moralista que más bien iba destinado a todos los adolescentes de la sala. Los jóvenes presentes escucharon el mensaje del hipnotizador sobre el respeto que le debían a sus padres y maestros, y fue con este mensaje, inculcado en el subconsciente de los hipnotizados, que el hipnotizador podría defenderse ante cualquier demanda por su abusiva intervención. Pero a pesar de que los adolescentes hipnotizados fueron los principales participantes en el evento, su participación fue 
semiconsciente $y$, en el momento en que afrontaron la necesidad de comprender lo que había pasado, lo único a lo que pudieron recurrir fue al relato de sus amigos. El posible aprendizaje que se daría a partir del evento dependería, primero, de la posibilidad de confiar en sus compañeros para creer en los relatos de lo sucedido. Como ha aseverado Michael Melbourne (2001): para entender y creer se requiere que el individuo confíe en lo que otros actores significativos dicen.

Lograr la confianza de otra persona es parte de un proceso fundamentalmente libre y que implica la participación activa de quien explica y de quien está ahí para comprender lo explicado.

A partir de ambas viñetas se entiende que los individuos pueden resistirse a comprender lo que se les pide porque primero han de comprender otras cosas necesarias para ellos, personal e individualmente. Cada persona suele hacer conexiones particulares de acuerdo a su edad o sus conocimientos previos, pero también acorde a la familiaridad que la persona siente respecto a la fuente de información. En muchos casos, lo que los individuos desean comprender puede ser algo totalmente inesperado-incluso para los mismos diseñadores de alguna exposición. El ejemplo claro es la situación en la que era importante denotar la diferencia entre lo real y lo irreal a un niño de cuatro años-aunque la escena que le aterrara fuera, a todas luces, un simulacro. Cuando un centro de ciencias no destina tiempo para el ensayo y la participación entonces es difícil asegurar que los niños entenderán lo planeado, y es posible que lo que entiendan sean otras cuestiones como el comportamiento rígido en salas de exhibición, la importancia de no preguntar para no parecer tonto o ignorante, la necesidad de permanecer callado para no ser reprimido.

Los procesos de comprensión varían en tiempo y es muy probable que entren en juego dos acepciones del verbo confiar. En inglés es posible distinguir claramente los significados de las palabras trust y self-confidence. En castellano, la palabra confianza se usa tanto para hablar del fiarse moralmente en alguien más, como de la acción de creer en uno mismo. Al respecto, creo que puedo afirmar que fui testigo de cómo Ismael desarrollaba confianza en el otro (nosotros los adultos) y en sí mismo, en paralelo. En cuanto a los adolescentes, no me atrevería a ahondar en la posibilidad de que hayan despertado del trance con su autoconfianza mermada; sin embargo, considero que Ismael tuvo la oportunidad de prepararse cognitivamente para comprender algo nuevo, a diferencia de los adolescentes.

En el primer caso, algo realmente crucial para generar la comprensión del niño, afirmar su confianza y darle nuevo conocimiento, habría sido exhibir a un robot con lo mecanismos expuestos. Una importante lección de mecánica se perdió en el diseño de la violenta simulación del mundo jurásico. Sobre la sesión del hipnotizador, ya se ha dicho que además de una buena explicación de lo ocurrido, hizo falta un hipnotizador ético y un "guía" preparado para acompañar el proceso de comprensión.

Tanto los adolescentes hipnotizados como Ismael, primero tenían que lograr confiar y creer en lo que la gente les decía. Tras la abusiva interacción con el hipnotizador, el comprender y creer en la posibilidad de la hipnosis desembocó en un conflicto básico entre el confiar o el desconfiar; creer o no creer. La confianza en sus compañeros les 
sirvió para creer en lo que escucharon que había sucedido. No tanto para entender exactamente lo que sucedió ni cómo sucedió.

De manera que existe una diferencia importante entre los dos casos: Ismael pudo participar en una serie de dramas que se organizaron improvisadamente para que él perdiera su miedo al comprender la irrealidad de los dinosaurios. En contraste, los adolescentes, a pesar de que participaron, al hacerlo sin un registro consciente de los dramas, fueron despertados para creer en las evidencias y los argumentos de otros. La confianza y la posibilidad de creer en otros fueron dos elementos clave para que los jóvenes entendieran qué había sucedido en ese escenario. Participar, confiar y creer son verbos necesarios para comprender algo increíble o desconocido. Welbourne (2001) ha sugerido que para comprender y creer es necesario también confiar en que lo que se nos dice que es cierto. Los adolescentes tuvieron que creer en lo que les decían sus compañeros porque confiaban en quienes se los decía. Ismael, por el contrario estaba en pleno desarrollo de su confianza hacia los demás para poder creer en lo que le decían y finalmente atreverse personalmente a participar para creer. Comprender, para Ismael y los chicos, vendría después de confiar y creer. Así que participar no lo es todo; confiar en quien explica y confiar para comprender es trascendental. De manera que el explicar ha de ser visto como algo que, en términos sociales, implica reconocer la individualidad de las personas y proveer el tiempo necesario para vencer la resistencia a través de la participación de cada individuo. Por lo tanto la institución ha de estar preparada con recursos suficientes para proveer de otros medios para el entendimiento.

\section{EXTRAPOLAR: SOBRE LA DESCONFIANZA ORGANIZADA}

El tema de la confianza tiene varias aristas e implicaciones diversas para la cultura científica en general debido a que la confianza es también un valor central en el avance del desarrollo tecnocientífico contemporáneo. El asunto aquí tratado es, en primer lugar, un inconveniente didáctico al tratarse de dos eventos en los que emociones negativas fueron generadas con fines de exhibición: la explotación del suspenso y drama para recrear la naturaleza animal; el uso del miedo infantil para generar el interés en una era geológica; y la explotación del ridículo y la inconsciencia inocente para divertir a un público sin explicar, al menos, los usos psicológicos de la hipnosis. Mermar la confianza de un visitante en su posibilidad para comprender en un centro de ciencias es un problema importante.

Significativamente, el tema de la confianza ha sido abordado en la literatura sobre el estudio del quehacer de los científicos. La confianza, una capacidad humana fundamentalmente social y que lleva al individuo a superar su desconfianza natural, ha sido también la base para el crecimiento de las redes científicas. Diversos autores han explicado que la ciencia se hace principalmente al dudar, y al probar y comprobar (Chalmers 1999; Shapin 1996; Shapin and Schafer 1995; Pickstone 2001; Harvey 2004). Las comunidades científicas han desarrollado un sistema de arbitraje, una dependencia epistémica (Hardwig 1985) entre ellos. Un sistema basado en la referencia al trabajo de otros y la descripción detallada y el arbitraje ciego entre pares para, entre otras cosas, controlar la desconfianza (Latour 1987). Compartir 
información mientras se genera también confianza ubica al hacer ciencia en el campo de lo político y no sólo de lo cognitivo.

El salto entre la confianza descrita en las viñetas hacia el estudio de las comunidades científicas puede parecer injustificado. Es posible que las motivaciones para superar la desconfianza en el universo de la divulgación y el de las comunidades no parezcan comparables a primera vista. Sin embargo, desde mi perspectiva, se trata de un fenómeno del comportamiento humano que es central para el avance del conocimiento, sea entre niños preescolares y adolescentes o entre hombres y mujeres adultos trabajando como científicos. La confianza en ambos universos es epistemológicamente significativa y equiparable aunque la organización social alrededor de la desconfianza no sea tan impactante en el mundo de la divulgación y la educación, y sí lo sea en el de la academia.

Tal vez dos viñetas no basten para convencer a todos los diseñadores de salas museísticas ni a todos los divulgadores y educadores en las ciencias que es necesario considerar cómo invitan a sus escuchas a confiar en ellos y por lo tanto en sus mensajes. Aún así puedo afirmar que quien explica algo-incluido el conocimiento científico-no logrará el objetivo de la comprensión si no media la confianza, además de la existencia de pruebas, explicaciones convincentes, fundamentos, experimentos y claridad.

\section{COMENTARIOS FINALES}

Es muy probable que la provisión de tiempos y espacios para la generación de la confianza sea un elemento básico para promover realmente una cultura científica. Hemos de reconocer, sin embargo, que el ser conscientes de la necesidad de dar tiempos suficientes y permitir el libre desarrollo de la confianza sean elementos que se pasan por alto porque finalmente lo que se busca son indicadores, en el corto plazo, de comprensión y diversión.

La necesidad que tienen hoy los centros de ciencias y museos de ciencias por presentar exposiciones comunicativamente eficientes es totalmente comprensible si consideramos la imperante necesidad que existe de asegurar que los visitantes llevarán consigo algún conocimiento tras la visita al centro. Estas visitas se dan dos o tres veces en la vida, de manera que los centros de ciencias han de anticiparse y diseñar exposiciones intelectualmente atractivas. Sin embargo estamos ante una contradicción importante. Al fomentar la interactividad con exposiciones hands on, en muchos casos se está dando una visión digerida de lo que es el conocimiento científico. No se da la idea de un fenómeno cognitivo inacabado, en proceso, y progresivo como es el avance científico en realidad. De manera que, como se evidenció en el planetario estudiado, es muy posible que los centros de ciencias estén llevando al individuo a desarrollar reacciones negativas que más tienen que ver con la desconfianza, y a aprender cuestiones erróneas como la existencia actual de los dinosaurios. Es posible que esto esté sucediendo ante tres situaciones: la resistencia a modificar las representaciones de la ciencia como un producto acabado, y prístino; el pasar por alto en las exhibiciones que, en realidad, los procesos de investigación llevan a los científicos a conceder que su conocimiento está siempre en ciernes, es 
inacabado, imperfecto y perfectible en cada momento; y finalmente, al dar por hecho que, por tratarse de instituciones de divulgación, sus contenidos son automáticamente confiables.

A pesar de que la desconfianza es una actitud humana natural $y$, como se ha expuesto aquí, es parte de la justificación del arbitraje científico, cuando los individuos tienen tiempo suficiente para vencer la desconfianza, comprenden. Cuando no tenemos el tiempo suficiente para observar, dudar, probar, comprobar y confiar, es muy probable que no logremos a cabalidad la comprensión de lo que se nos explica porque tampoco hubo tiempo ni medió algún argumento explícito que apoyara la generación de la confianza. La comprensión profunda de alguna explicación científica es, tal vez, más improbable una vez que se ha fomentado una disposición inmediatista para comprender; es decir, se ha privilegiado el ritmo de lo impactante, de lo contundente, en las salas de ciencias en general. Es posible aniquilar la disciplina y la disposición de largo plazo necesaria para comprender cualquier cosa más allá de lo empírico o superficial ya que existe la posibilidad de que se esté supliendo esta disposición mental y social disciplinada y de largo plazo con exposiciones prefabricadas en las que presionar un botón y observar es lo único que se pide al visitante. Es loable que en centros de ciencias siga combinándose la explicación por impacto y la sostenida a lo largo de exposiciones temáticas. Sin embargo, la sugerencia es que no se tome a la ligera la recurrente necesidad histórica de regenerar la legitimidad de las ciencias y sus instituciones.

Aquí se ha sugerido que la interacción basada en la confianza es un elemento necesario para la divulgación del conocimiento científico, lo mismo que el tiempo y espacio definidos para la interacción de los individuos entre sí y no sólo con los objetos. Así como las comunidades científicas logran sus avances a lo largo del tiempo, procesualmente, promoviendo la participación de los individuos para la comprensión de los fenómenos y para la generación de confianza en los resultados propios y de otros equipos e individuos, aquí se ha propuesto que se entienda la acción de comprender no como un proceso ciego, ni como una cuestión de fe. La confianza en la ciencia no es perenne y esto se ha de destacar en la planeación de salas en centros de ciencias. Comprender es un proceso que oscila entre la confianza y la desconfianza, de modo que el tiempo para lograr la comprensión y la confianza se ha de proveer. En otras palabras, un centro de ciencias ha de estar preparado para enfrentar la desconfianza natural del ser humano.

Este análisis muestra que un centro de ciencias ha de proveer los elementos que permitan confiar en él y su personal, además de permitir el espacio, los diseños y los tiempos necesarios para entender conocimientos científicos. Se puede argumentar también que exposiciones no confiables generen la necesidad de ir más a fondo en los fenómenos hasta cultivar una disciplina similar a la que caracteriza al quehacer científico; sin embargo, considero que es más adecuado, moral, política y pedagógicamente, aprender a generar confianza cuando se trabaja con niños y adolescentes. 


\section{SUGERENCIAS DE SOLUCIÓN}

En este estudio se tomó como referente analítico el conocimiento que existe sobre la importancia de la confianza en el avance de las comunidades científicas. A partir de ese referente es posible pensar que para que un centro de ciencias contribuya en la promoción del conocimiento científico en la sociedad es ideal que se generen espacios de aprendizaje que asimilen las formas y los procesos de trabajo científico además del conocimiento que las ciencias han puesto en circulación. De manera que una sugerencia es transformar cada centro de ciencias en un centro de investigación además de centro de exhibición: Promover la participación del personal de estas instituciones en investigaciones sobre la comunicación de la ciencia y el desarrollo de exhibiciones propias. Complementar así la muy socorrida compra de exhibiciones prefabricadas hechos por centros donde sí hay desarrollo propio-instituciones que han globalizado el modelo de centro de ciencias que hoy predomina-para crear espacios de generación de conocimiento en cada centro de ciencias o planetario. Es muy probable que la participación en este tipo de espacios provea al personal que está en contacto con el público con nociones mejor fundamentadas de su papel en la divulgación de las ciencias y las implicaciones de tiene su desempeño a favor de una cultura científica robusta. Finalmente, dudo que esté de más recordar que la confianza en la ciencia no ha sido, históricamente, instantánea; de manera que difícilmente será infinita, uniforme o total, aunque en ocasiones el tema se tome a la ligera.

\section{REFERENCIAS}

Briggs, J.L. (1998). Innuit morality play: the emotional education of a three-year-old, New Haven and London: Yale University Press.

Chalmers, A.F. (1999). What is this thing called science?, tercera edición, Reino Unido: Open University Press.

Fierro Gossman, J. (2001). "La divulgación de la ciencia" en Antología de la divulgación de la ciencia en México, J. Tonda, A. M. Sánchez y N. Chávez (coord.), Col. Divulgación para Divulgadores, México: Dirección General de Divulgación de la Ciencia y UNAM, pp. 158-168.

Hardwig, J. (1985). "Epistemic dependence", en Journal of Philosophy, 82: 145-162.

Harvey, P. (2004). Arresting mobility or locating expertise: "Globalisation" and the "Knowledge society", artículo presentado en el taller "Mobilising structures, structures of mobility, globalization and the power of place", Dubrovnik.

Latour, B. (1987). Science in action: How to follow scientists and engineers through society, Cambridge, Mass.: Harvard University Press.

Pickstone, J. V. (2001). Ways of knowing. A new history of science, technology and medicine, Manchester: Manchester University Press.

Sánchez Vázquez, M. A. (2005). Scientific indifference: understanding science in a Mexican planetarium, Universidad de Manchester, Departamento de Antropología Social, tesis doctoral. 
Shapin, S. (1996). The scientific revolution, Chicago y Londres: The University of Chicago Press.

Shapin, S. y Schaffer, S. (1985). Leviathan and the air-pump: Hobbes, Boyle and the experimental life, Princeton: Princeton University Press.

Toren, C. (1993). "Making history: The significance of childhood cognition for a comparative anthropology of mind", en Man, vol. 28, núm. 3, pp. 461-478.

Waterfield, R. (2002) Hidden depths: The story of hypnosis, Londres: Macmillan Press.

Welbourne, M. (2001). Knowledge, Central Problems of Philosophy Series, Reino Unido: Acumen Publishing Limited. 


\section{SUMMARY}

The author describes two events that took place in a science centre in a Mexican city. The analysis invites the reader to reflect upon the need to generate confidence among the visitors of those institutions dedicated to the popularization of scientific knowledge. The processes through which a preschooler and a group of teenagers understood the knowledge presented to them in emotive and significant situations are described. It is explained how fear and distrust hampered the communication of scientific knowledge. It is suggested that trusting in the people who give out scientific explanations constitutes an ethical and political resource required to foster a scientific culture centered on the understanding of the processes of knowledge production.

Key words: Ethnography; science centres; understanding; trust; popularization; public communication of science.

1 Una primera versión de este artículo fue propuesta para ser presentada en la X Reunión de la RED POP celebrada del 9 al 11 de mayo del 2007 en San José, Costa Rica.

$\underline{2}$ La autora desea agradecer a los dos lectores anónimos de Revista Eureka sobre Enseñana y Divulgación de las Ciencias, quienes con sus valiosos comentarios a la primera versión del artículo, contribuyeron a su mejora significativa. 